Nazarii Kobyliak ${ }^{1}$, Ludovico Abenavoli ${ }^{2}$, Galyna Mykhalchyshyn ${ }^{1}$, Tetyana Falalyeyeva ${ }^{3}$, Olena Tsyryuk ${ }^{3}$, Liudmyla Kononenko ${ }^{1}$, Dmytro Kyriienko1, 4 , luliia Komisarenko ${ }^{1}$

${ }^{1}$ Department of Endocrinology, Bogomolets National Medical University, Kiev, Ukraine

${ }^{3}$ Taras Shevchenko National University of Kyiv, Kyiv, Ukraine

${ }^{4}$ Kyiv City Clinical Endocrinology Center, Kiev, Ukraine

\title{
Probiotics and smectite absorbent gel formulation reduce liver stiffness, transaminase and cytokine levels in NAFLD associated with type 2 diabetes: a randomized clinical study
}

\section{ABSTRACT}

Introduction. In double-blind single center randomized clinical trial (RCT), the efficacy of alive probiotics supplementation with smectite gel vs. placebo in type 2 diabetes patient with non-alcoholic fatty liver disease (NAFLD) detected on ultrasonography (US) were studied.

Material and methods. A total of 50 patients met the criteria for inclusion. They were randomly assigned to receive Symbiter Forte combination of probiotic biomass with smectite gel $(250 \mathrm{mg}$ ) or placebo for 8-weeks. The primary main outcomes were the change in fatty liver index (FLI) and liver stiffness (LS) measured by shear wave elastography (SWE). Secondary outcomes were the changes in transaminases activity, serum lipids and cytokines levels.

Results. All subjects completed the study and received more than $90 \%$ of prescribed sachets. In respect to

Address for correspondence:

Nazarii Kobyliak, PhD

Assistant of Endocrinology Department,

Bogomolets National Medical University

Kyiv, 01601, Pushkinska 22a str., Ukraine

Phone: +380442356005

e-mail: nazariikobyliak@gmail.com

Clinical Diabetology 2019, 8, 4, 205-214

DOI: $10.5603 / D K .2019 .0016$

Received: 25.03.2019

Accepted: 25.04.2019 our primary endpoints, FLI and LS insignificant decrease in both interventional and placebo groups. However, when we compare mean changes across groups from baseline, expressed in absolute values, the reduction of both LS $(-0.254 \pm 0.85$ vs. $0.262 \pm$ $\pm 0.77 ; p=0.031$ ) were observed. Analysis of secondary outcomes showed that co-administration of probiotic with smectite lead to significant reduction of alanine aminotransferase (ALT), aspartate aminotransferase (AST), total cholesterol, IL-1 $\beta$, and tumor necrosis factor (TNF- $\alpha$ ) after 8 weeks.

Conclusion. In this RCT, we confirmed previously reported animal data, showing that co-administration of probiotic with smectite manifested with reduction of LS, liver transaminases and chronic systemic inflammation. (Clin Diabetol 2019; 8, 4: 205-214)

Key words: diosmectite, nutraceuticals, non-alcoholic fatty liver disease, probiotics, Lactobacillus, Bifidobacterium, Propionibacterium

\section{Introduction}

Non-alcoholic fatty liver disease (NAFLD) is an unique term that combines several components, in particular the simple steatosis with excessive intra hepatic fat accumulation over $5 \%$ of organ weight, non-alcoholic steatohepatitis (NASH), constituted by steatosis with development of necroinflammation, 
fibrosis and finally cirrhosis and hepatocellular carcinoma [1]. NAFLD is now recognized as the hepatic manifestation of metabolic syndrome and often associated with many pathologies such as type 2 diabetes (T2D), visceral obesity and insulin resistance (IR) [2] Globally, the incidence of NAFLD is recently growing and has reached in Western Countries $20-30 \%$ and $5-18 \%$ in Asia [3]. Epidemiology data supports the idea that NAFLD is the most common reason of chronic liver disease, major cause of liver-related morbidity and mortality and urgent problem for global public health $[4,5]$.

Currently, the treatment of NAFLD is based on lifestyle changes, included enhancement of daily physical activity parallel with calorie restriction [2]. New therapeutic approaches have been under study in pre-clinical and clinical studies. Nowadays, modulation with gut microbiota composition and direct its metabolic effects in a perspective of prevention or treatment of NAFLD [6]. Among the suggested strategies, probiotic supplementation, as the intake of microorganisms capable when administered in acceptable amounts, confers a health benefit on the host [7]. The positive impact of Lactobacilli and Bifidobacteria, the most studied and commonly used probiotic strains in the treatment and prevention of obesity-associated disorders, have been previously discussed in the literature [8]. Moreover, several potential bacterial candidates, such as Saccharomyces cerevisiae var. boulardii, Parabacteroides goldsteinii, Enterobacter halii or Akkermansia muciniphila, have been identified and innovative mechanisms of action overriding their beneficial effects for obesity have been elucidated $[9,10]$. In this regard, many researchers are trying to describe the role played by the different bacterial strains on the NAFLD management. Our choice of bacteria formulation for the present investigation was based on previous comparative preclinical studies of different probiotic strains intended for treatment and prevention of NAFLD and obesity [11, 12]. Intervention with poly-probiotic mixtures containing both alive and lyophilized strains led to significant reduction of total and visceral adipose tissue weight, steatosis, and necroinflammation, and to enhanced insulin sensitivity in rats with monosodium-glutamate (MSG) induced obesity model [11-13].

Smectite is a natural silicate clay belonging to the dioctahedral smectite class, binds to intestinal mucous, forms multilayer structure with high plastic viscosity and powerful coating properties hence preserving integrity of the mucus, and has the ability to absorb directly bacterial toxins, bacteria, viruses and bile salts $[14,15]$. Diosmectite also has a protective effect against intestinal inflammation [16] hence suppressing production of cytokines such as interleukin-8 from secretory epithelial cells [17] and to attenuating the proinflammatory action of tumor necrosis factor- $\alpha$ (TNF- $\alpha$ ) [18]. We proposed that all of these pharmacological properties may be beneficial for the treatment of NAFLD.

Based on preclinical data, in rats with MSG-induced obesity supplementation of alive probiotics with smectite gel (Symbiter-Forte formulation) significant reduce chronic systemic inflammatory markers (IL-1 $\beta$, TNF- $\alpha$ ) [19], total NAS (NAFLD activity score) score, with marked decrease of lobular inflammation $(0.13 \pm 0.09$ vs. $0.33 \pm 0.15)$ as compared to administration of probiotic alone [20].

Current aims were to provide single center randomized clinical trial (RCT) with double-blind fashion to assess the efficacy of alive probiotics combination with smectite gel (Symbiter-Forte) vs. placebo in T2D patient with NAFLD detected on ultrasonography (US).

\section{Material and methods}

The recruitment started after the approval of RCT protocol by local commission of bioethics in Kyiv City Clinical Endocrinology Center. All procedures, associated with RCT, were in agreement with the guidelines of the 1975 Declaration of Helsinki. Before study began all participants gave written informed consent and were fully explained with purpose and its methodology.

\section{Study design}

This study was registered at ClinicalTrials.gov under identifier - NCT03614039 as single-center placebo controlled, double-blind, parallel group study with inclusion of 51 T2D patients. Participants were randomized by the study statistician based on computer-generated list to intervention (Symbiter Forte) or placebo group. The allocation of groups was blind to investigators and patients. Moreover, to maintain blind and parallel study fashion the statistician was not aware of the allocation of participants to intervention. Treatment period continued for 8 weeks. Both placebo and intervention were administered as a sachet formulation with similar organoleptic characteristics (e.g., taste and appearance). The Symbiter Forte was supplied by Scientific and Production Company " "O.D. Prolisok" (Ukraine). It contains combination of smectite gel $(250 \mathrm{mg})$, supplemented with biomass of 14 alive probiotic strains: Bifidobacterium $\left(1 \times 10^{10}\right.$ colony forming units - CFU/g), Lactobacillus + Lactococcus $\left(6 \times 10^{10} \mathrm{CFU} / \mathrm{g}\right)$, Acetobacter $\left(1 \times 10^{6} \mathrm{CFU} / \mathrm{g}\right)$ and short-chain fatty acids producing Propionibacterium (3 $\times 10^{10} \mathrm{CFU} / \mathrm{g}$ ) genera. Over a treatment period the participants received 1 sachet $(10 \mathrm{~g})$ of probiotic-smectite or placebo per day. 
For minimization of the dietary changes effects, 2 weeks before first sachet prescribed, all patients were instructed in one-on-one sessions with a qualified dietitian to follow a therapeutic lifestyle-change diet as classified by the National Cholesterol Education Program (NCEP). In addition, participants were instructed to continue with stable anti-diabetic drugs and received standardized slight physical exercise for 1 hour per day.

Throughout the study, weekly phone follow-up visits were provided for assessment of compliance, adherence to the protocol, as well as the recording of adverse events. The effectiveness of therapy was compared and evaluated separately in the two groups.

\section{Inclusion and exclusion criteria}

Current study has similar design to previously reported by our group RCTs were effects of alive probiotic alone or in combination with omega-3 fatty acids in NAFLD were assessed [21, 22]. The main inclusion criteria were: adult T2D patients treated with diet and exercise alone or metformin, SUs and insulin at stable dose at least 4 weeks prior to randomization; body mass index $(\mathrm{BMI}) \geq 25 \mathrm{~kg} / \mathrm{m}^{2}$; presence of NAFLD detected on US as previously described [21, 22]; aspartate transaminase (AST) and alanine transaminase (ALT) $\leq 3$ $\times$ upper limit of normal.

The main exclusion criteria were decompensated liver disease including ascites, encephalopathy or variceal bleeding or presence of other chronic diffuse liver diseases such as chronic viral hepatitis associated with HBV, HCV or HEV-infection; drug-induced liver disease; hereditary deficiency of antitrypsin-1, Wilson's disease or idiopathic hemochromatosis. Patients with history over a two-year period or with active alcohol abuse which defined as consumption more than 2 standard drinks (> $20 \mathrm{~g} /$ day) for women and 3 standard drinks (> $30 \mathrm{~g} / \mathrm{d}$ ) for men were also excluded. Treatment within 3 months prior to randomization with agents that can impact microbiota composition such as probiotic, prebiotic or antibiotic; vitamin E, omega-3 fatty acids or medications with evidence for effects on NAFLD (pioglitazone, glucagon-like peptide-1 [GLP-1] analogues, dipeptidyl peptidase IV inhibitors, ursodeoxycholic acid) [21, 22]. Presence of uncontrolled cardiovascular or respiratory disease, active malignancy, or chronic infections.

\section{Outcomes assessment}

The primary main outcomes were the changes in fatty liver index (FLI) and liver stiffness (LS) measured by Shear Wave Elastography (SWE). The methodology of outcomes assessment was previously described $[21,22]$.
LS was measured by SWE using a multifrequency convex transducer (2-5 MHz) and Ultima PA ultrasound equipment (Radmir, Ukraine). Firstly, in B-mode we estimated position of liver (the ratio of the edge to the costal arch, the availability of acoustic windows) investigated both lobes of the liver and carried out their antero-posterior size biometrics on inspiration. Even or uneven contour of the liver was assessed, as well as acute or rounded front-bottom corner of the liver. We evaluated the echogenicity (normal, low or high) and echostructure (fine particles - 1-2 $\mathrm{mm}$, medium particles - 3-4 $\mathrm{mm}$, and coarse particles - more than $5 \mathrm{~mm}$ ). Sound conductivity of the liver parenchyma or opposite US attenuation in the front-rear direction of liver was evaluated by Hamaguchi's B-mode criteria $[21,22]$.

SWE was carried out by the standard algorithm for 2D SWE. Especially carefully navigated region of interests (ROI) and SV of 2D SWE by B-mode and removed SWE artifacts. We performed 10 valid measurements of LS in every patient, and a median value was calculated, the result being measured in $\mathrm{kPa}[21,22]$.

$\mathrm{FLI}$ a validated prediction score for hepatic steatosis severity designed Bedogni et al [23]. FLI was calculated based on laboratory and anthropometric measures, including triglycerides, gamma glutamyl transferase (GGT), BMI, and waist circumference (WC), by using the following formula:

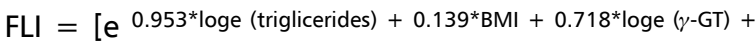
$+0.053^{*}$ waist circumference -15.745$) /\left(1+\mathrm{e}^{0.953^{*} \text { loge (triglicerides) }}\right.$ $+0,139^{*} \mathrm{BMI}+0.718^{*} \operatorname{loge}(\gamma-\mathrm{GT})+0.053^{*}$ waist circumference -15.745$\left.)\right]$ $\times 100$
}

Secondary outcomes were the changes in transaminases activity, serum lipids and cytokines (TNF- $\alpha$, interleukin [IL]-1 $\beta$, IL-6, IL-8, and interferon [IFN]- $\gamma$ ) levels. All values were determined following a 12-h fasting period, by the hospital clinical laboratory.

Anthropometric data including weight and height were measured to an accuracy of $0.1 \mathrm{~kg}$ and $0.5 \mathrm{~cm}$, respectively. BMI was calculated as body weight in kilograms divided by the square of the participant's height in meters (weight/height ${ }^{2}$ ). Waist circumference (WC, narrowest diameter between xiphoid process and iliac crest) was measured as well [21, 22].

Activity of ALT and AST in serum were determined by the standard biochemical methods. Serum concentrations of total cholesterol (TC), high-density lipoprotein cholesterol (HDL-C) and triglyceride (TG) concentrations were measured using the standard enzymatic methods with commercially available kits (BioVendor, Czech Republic). Low-density lipoprotein 
Table 1. Anthropometric, clinical and laboratory parameters in examined patients (M \pm SD or \%)

\begin{tabular}{|c|c|c|c|}
\hline Parameters & Placebo group $(n=24)$ & Probiotic-smectite group $(n=26)$ & $\mathbf{P}$ \\
\hline Age (years) & $57.38 \pm 9.92$ & $53.23 \pm 10.09$ & 0.150 \\
\hline Duration of T2D (years) & $5.33 \pm 2.82$ & $6.46 \pm 5.92$ & 0.401 \\
\hline Metformin, \% (n) & 71.4 & 70.0 & 0.905 \\
\hline Sulfonilureas, \% (n) & 53.6 & 43.3 & 0.436 \\
\hline Insulinotherapy, \% (n) & 25.0 & 36.7 & 0.337 \\
\hline BMI $\left[\mathrm{kg} / \mathrm{m}^{2}\right]$ & $32.55 \pm 3.62$ & $33.19 \pm 4.93$ & 0.601 \\
\hline Weight [kg] & $92.3 \pm 11.49$ & $94.81 \pm 12.04$ & 0.455 \\
\hline Waist circumference $[\mathrm{cm}]$ & $94.08 \pm 4.96$ & $96.57 \pm 5.01$ & 0.084 \\
\hline FLI & $80.16 \pm 10.36$ & $82.11 \pm 10.95$ & 0.521 \\
\hline LS [kPa] & $7.69 \pm 1.33$ & $8.02 \pm 1.39$ & 0.401 \\
\hline ALT [IU/L] & $35.93 \pm 16.32$ & $35.88 \pm 16.89$ & 0.991 \\
\hline AST [IU/L] & $36.96 \pm 17.66$ & $33.31 \pm 13.94$ & 0.420 \\
\hline$\gamma$-GT [IU/L] & $45.33 \pm 12.21$ & $47.87 \pm 21.84$ & 0.088 \\
\hline $\mathrm{TC}[\mathrm{mmol} / \mathrm{L}]$ & $5.92 \pm 0.8$ & $6.15 \pm 0.83$ & 0.320 \\
\hline TG $[\mathrm{mmol} / \mathrm{L}]$ & $2.50 \pm 0,91$ & $2,53 \pm 0,99$ & 0.888 \\
\hline $\mathrm{HDL}-\mathrm{C}[\mathrm{mmol} / \mathrm{L}]$ & $1.4 \pm 0.27$ & $1.33 \pm 0.23$ & 0.292 \\
\hline LDL-C [mmol/L] & $3.41 \pm 0.68$ & $3.64 \pm 0.77$ & 0.270 \\
\hline TNF- $\alpha[\mathrm{pg} / \mathrm{mL}]$ & $50.52 \pm 18.73$ & $51.57 \pm 20.35$ & 0.586 \\
\hline $\mathrm{IL}-1 \beta[\mathrm{pg} / \mathrm{mL}]$ & $47.69 \pm 21.36$ & $44.49 \pm 19.88$ & 0.850 \\
\hline IL-6 [pg/mL] & $13.89 \pm 8.80$ & $13.22 \pm 8.40$ & 0.785 \\
\hline IL-8 [pg/mL] & $26.05 \pm 8.27$ & $29.25 \pm 8.04$ & 0.785 \\
\hline$\gamma$-INF & $168.29 \pm 75.51$ & $187.23 \pm 75.53$ & 0.380 \\
\hline
\end{tabular}

cholesterol (LDL-C) concentration was calculated using the Friedewald equation [24].

The contents of serum interleukins (TNF- $\alpha$, IL- $1 \beta$, IL-6, IL-8, and IFN- $\gamma$ ) were measured by enzyme-linked immunosorbent assay (ELISA) method. The studied cytokines were immobilized in 96-well plates with adsorption surface. They were added with primary and secondary enzyme-labeled antibodies (Sigma). An appropriate substrate was then added and produced a detectable product in the enzymatic reaction. The optical densities of the colored solutions in wells immediately after the enzymatic reaction termination depicted the level of cytokines of the different group.

\section{Statistical analysis}

The SPSS statistical package, version 20.0 (SPSS, Inc., Chicago, Illinois) and GraphPad Prism, version 6.0 (GraphPad Software, Inc., La Jolla, CA, USA) were used for all statistical analyses and a $P$ value less than 0.05 was considered statistically significant. All data in this study were expressed as mean \pm standard deviation (M $\pm \mathrm{SD}$ ) or \%. Data distribution was analyzed using the Kolmogorov-Smirnov normality test. The baseline characteristics of participants in the 2 groups were compared using independent sample t-tests and chi-squared $\left(\chi^{2}\right)$ test. The changes in outcomes of the participants after the initiation of therapy and end of the trial were compared by paired sample $t$-tests. Analysis of covariance (ANCOVA) was used to identify any differences between the 2 groups after intervention, adjusting for baseline measurements and confounders (BMI and sex).

\section{Results}

In this single-center RCT, a totally 51 patients with NAFLD and T2D were randomly allocated to receive probiotic additionally supplemented with smectite (Symbiter Forte group, $n=26)$ or placebo $(n=25)$ for 8 weeks, respectively. The groups were homogeneous according to age, sex and diagnostic criteria (Table 1). One patient from placebo group did not complete study due to lost of follow up. In final intention to treat analysis 50 patients were included which received more than $90 \%$ of prescribed sachets formulation in double-blind treatment. The compliance rate was comparable between groups - 91.7\% in placebo and $92.3 \%$ in Symbiter Forte group respectively ( $p=$ 0.993). Patients were satisfied with the organoleptic properties of both nutraceuticals formulation. During study period probiotic-smectite and placebo were well tolerated. Across the study patients complained only with several mostly gastrointestinal symptoms. 


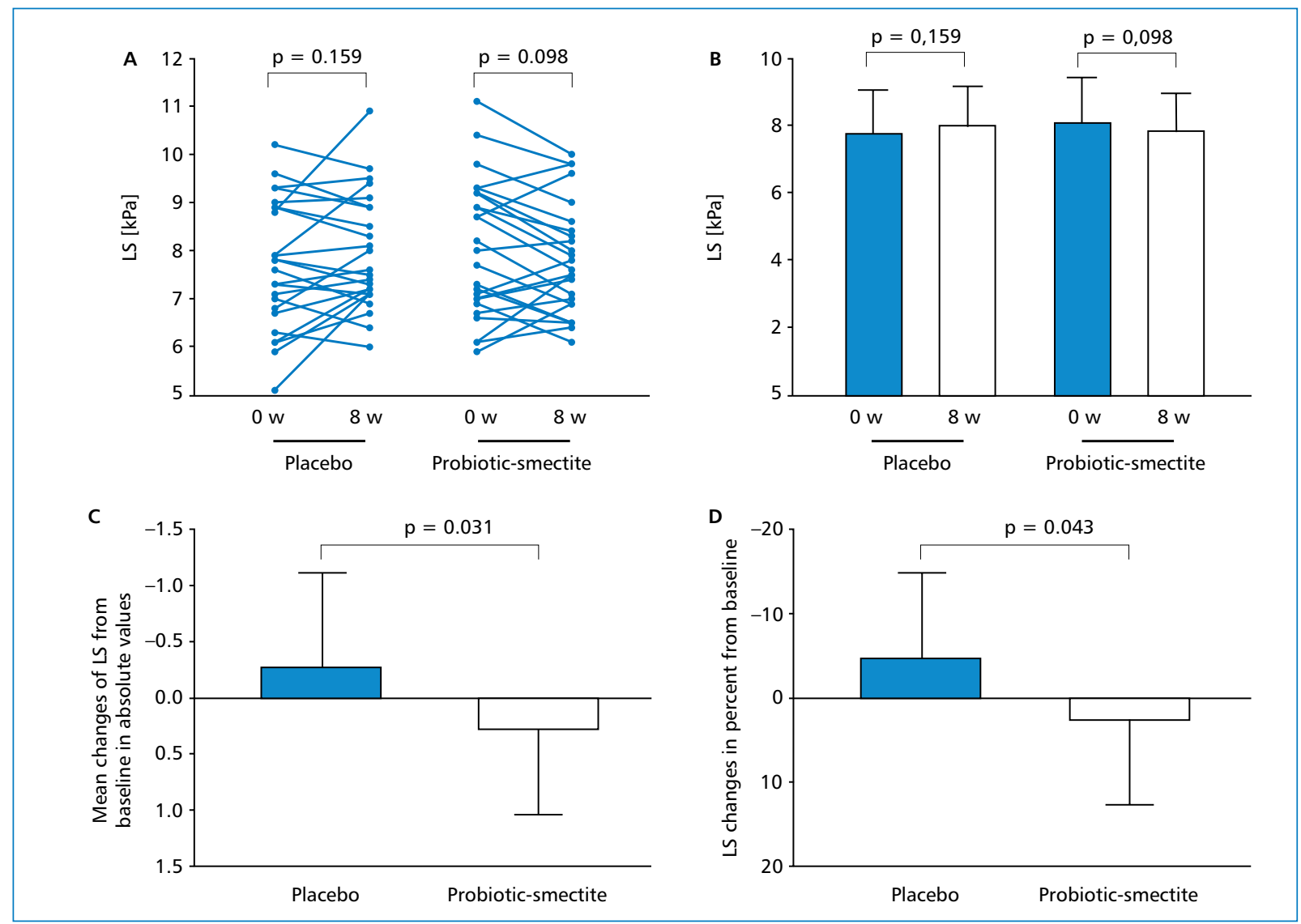

Figure 1. Primary outcomes analysis with accent on LS changes. A, B - intra-group analysis of changes at baseline and after interventon. Data expressed in mean \pm SD $(A)$ and individual values at baseline and after 8 weeks of treatment; C, D - analysis of inter-group mean changes of absolute values (C) or percentages (D) from baseline to end of treatment throughout the study. ANCOVA was used to identify any differences between the 2 groups after intervention

All adverse events were mild, reversed spontaneously and did not lead to participants withdrawn from the study. Patients received placebo reported adverse events were nausea ( $n=2 ; 8.33 \%$ ), mild abdominal pain $(n=1 ; 4.16 \%)$ and short-term diarrhea $(n=2$; $8.33 \%)$. The main complaints in Symbiter Forte group were short-term diarrhea $(n=2 ; 7.69 \%)$ and constipation ( $n=1 ; 3.84 \%)$, heartburn ( $n=1 ; 3.84 \%$ ), mild abdominal pain ( $\mathrm{n}=1 ; 3.84 \%$ ) and dizziness ( $n=1 ; 3.84 \%$ ). The total prevalence of adverse events was comparable between groups (placebo $=20.8 \%$ vs. probiotics-smectite $=23.1 \%, p=0.848$ ) .

There were no significant differences between the groups at baseline in terms of age, sex, diabetes duration, anthropometric and laboratory measurements (Table 1). Participants were treated with oral anti-diabetic agents, insulin or their combination. Recent study suggested that metformin, GLP-1, DPP-4 inhibitors are known to has pleiotropic effects beyond glucose reduction, including improvement of lipid profiles, bile acids and finally gut microbiota $[25,26]$. To elude possible interaction between incretin-mimetics and gut microbiota, patients treated with these class of anti-diabetic drugs were excluded from the study. From the other hand metformin nowadays recognized as first line therapy in patients with T2D, so it would be unethical to exclude metformin from participant therapy regimen. In our study to avoid this bias we randomized equal portions of patients, treated with stable doses of metformin at least 4 weeks prior to study start. In general, at baseline proportion of patients on insulintherapy $(p=0.337)$, treated with sulfonylureas ( $p=$ $0.436)$ and/or metformin ( $p=0.905)$ were comparable between the groups (Table 1 ).

Primary outcome changes dynamics from baseline to 8 weeks after intervention are presented in Figures 1 and 2. Both our primary endpoints the LS measured by SWE and FLI insignificant decrease after probioticsmectite treatment and increase in placebo groups (Figures 1A, B; 2A, B). However, we observed significant differences between mean changes of LS expressed in absolute value $(-0.254 \pm 0.85$ vs. $0.262 \pm 0.77$; $\mathrm{p}=0.031)$ or percentages $(-4.427 \pm 12.6$ vs. $2.38 \pm$ $10.25 ; p=0.043$ ) from baseline to end of treatment in 


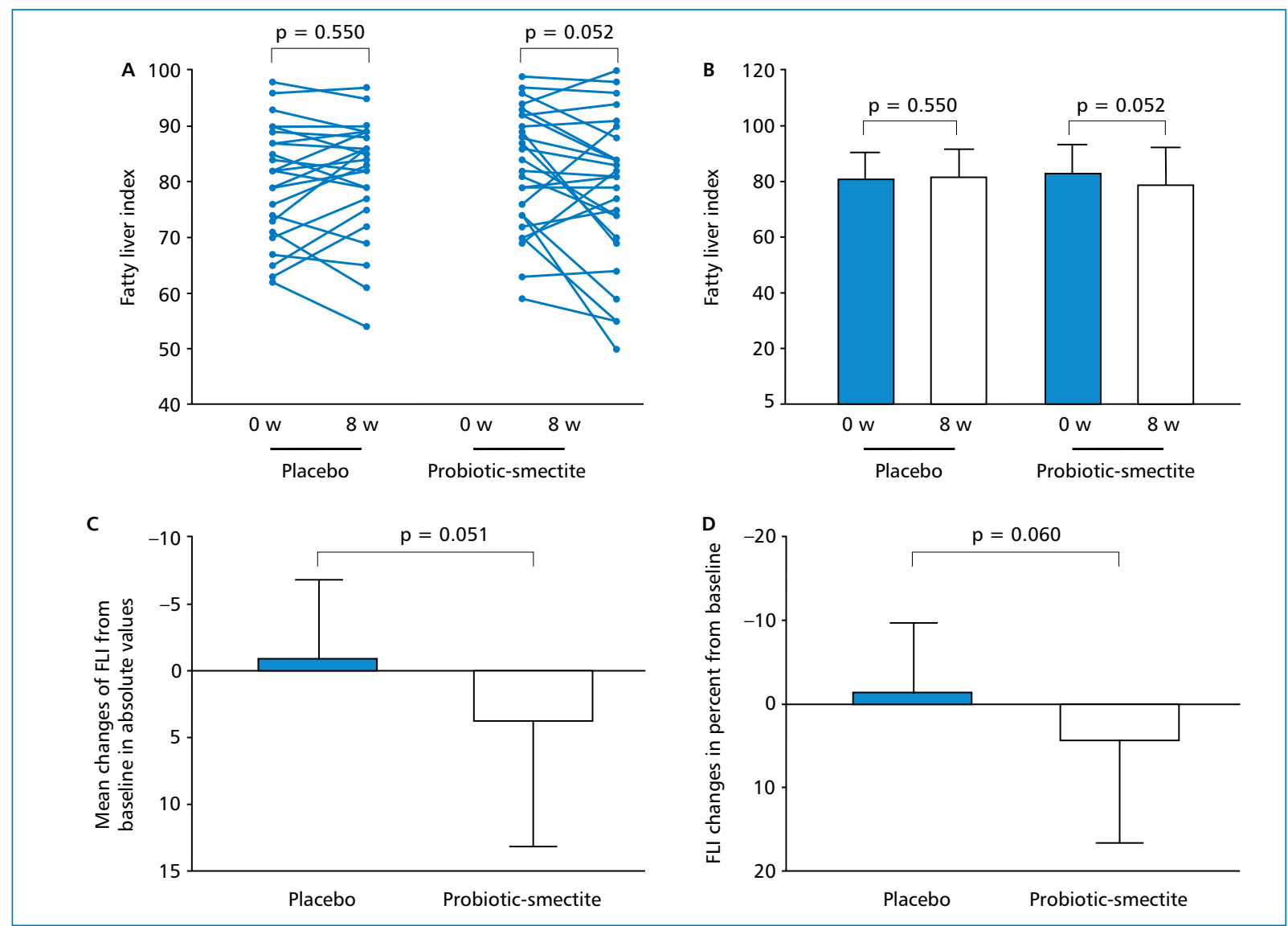

Figure 2. Primary outcomes analysis with accent on FLI changes. A, B - intra-group analysis of changes at baseline and after interventon. Data expreesed in mean \pm SD $(A)$ and individual values at baseline and after 8 weeks of treatment; C, D - analysis of inter-group mean changes of absolute values (C) or percentages (D) from baseline to end of treatment throughout the study. ANCOVA was used to identify any differences between the 2 groups after intervention

ANCOVA analysis (Figures 1C, D). In respect to another our primary endpoints, FLI mean changes across groups from baseline, expressed in absolute values $(-0.750$ \pm 1.23 vs. $3.769 \pm 1.84 ; p=0.051)$ or percentages $(-1.194 \pm 8.43$ vs. $4.471 \pm 12.15 ; p=0.06)$ were insignificant (Figures 2C, D).

Analysis of secondary outcomes showed that co-administration of probiotic with smectite lead to significant reduction of ALT (35.88 \pm 16.89 vs. 29.25 $\pm 10.48 ; p=0.016)$, AST $(33.31 \pm 13.94$ vs. $30.0 \pm$ $11.67 ; \mathrm{p}=0.021), \mathrm{TC}(6.15 \pm 0.83$ vs. $5.86 \pm 0.81$; $\mathrm{p}=0.010), \mathrm{IL}-1 \beta$ (44.49 \pm 19.88 vs. $37.75 \pm 14.02$; $\mathrm{p}=0.037)$ and TNF- $\alpha(51.57 \pm 20.35$ vs. $44.81 \pm 18.14$; $\mathrm{p}<0.001$ ) as compared to week 8 (Figures 3-5, Table 2). However, in between groups analysis changes remained significant only ALT expressed in absolute values ( $p=0.022$, Table 2 ). In the placebo group, changes were insignificant for all parameters which were included in secondary outcomes analysis.

\section{Discussion}

In this single-center RCT, it has been demonstrated that probiotic additionally supplemented with smectite (Symbiter Forte) insignificantly decreased both FLI and LS values measured by SWE in primary outcomes analysis. Analysis of secondary outcomes showed significant reduction of transaminases activity, total cholesterol, IL- $1 \beta$ and TNF- $\alpha$ values in intervention as compared to placebo group after 8 weeks of treatment. However, in between group ANCOVA analysis were mean values from baseline were analyzed, changes remained significant only for LS and ALT activity.

Mofidi et al. [27], similar to the present study, used LS with transient elastography (FibroScan ${ }^{\circledR}$ ) and hepatic steatosis (CAP score) measurement, to evaluate the efficacy of synbiotic supplementation in lean NAFLD patients. In the randomized, double-blind, placebo-controlled, clinical trial hepatic steatosis and fibrosis reduction was observed in both groups; how- 


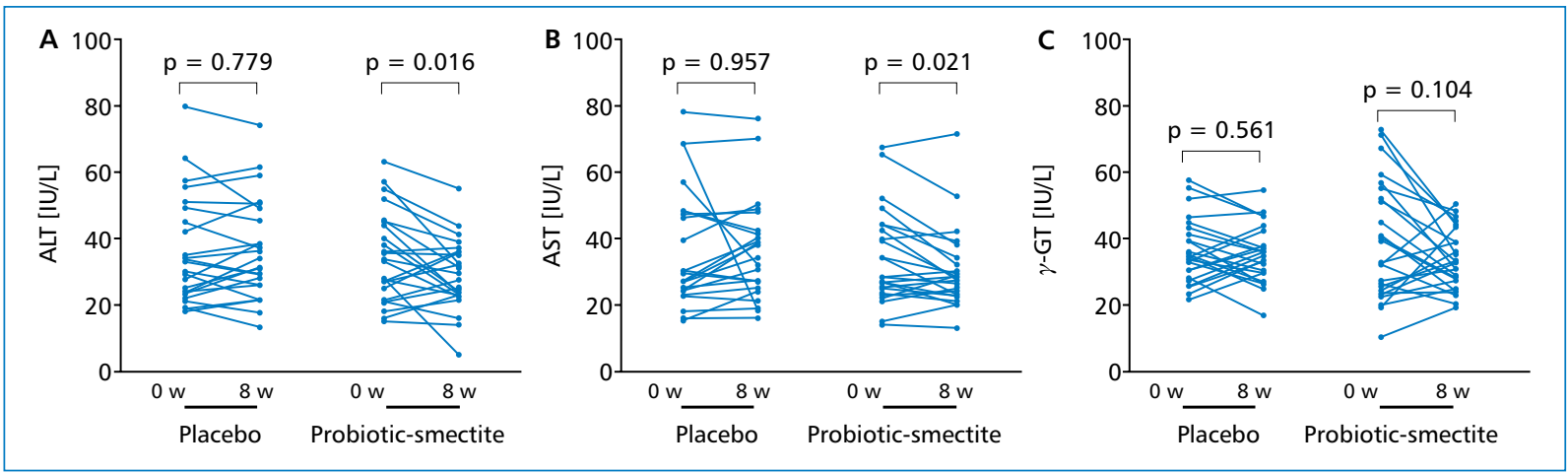

Figure 3. Secondary outcomes analysis with accent on transaminases changes. A, B, C - intra group analysis of changes at baseline and after interventon. Data expreesed as individual values at baseline and 8-week

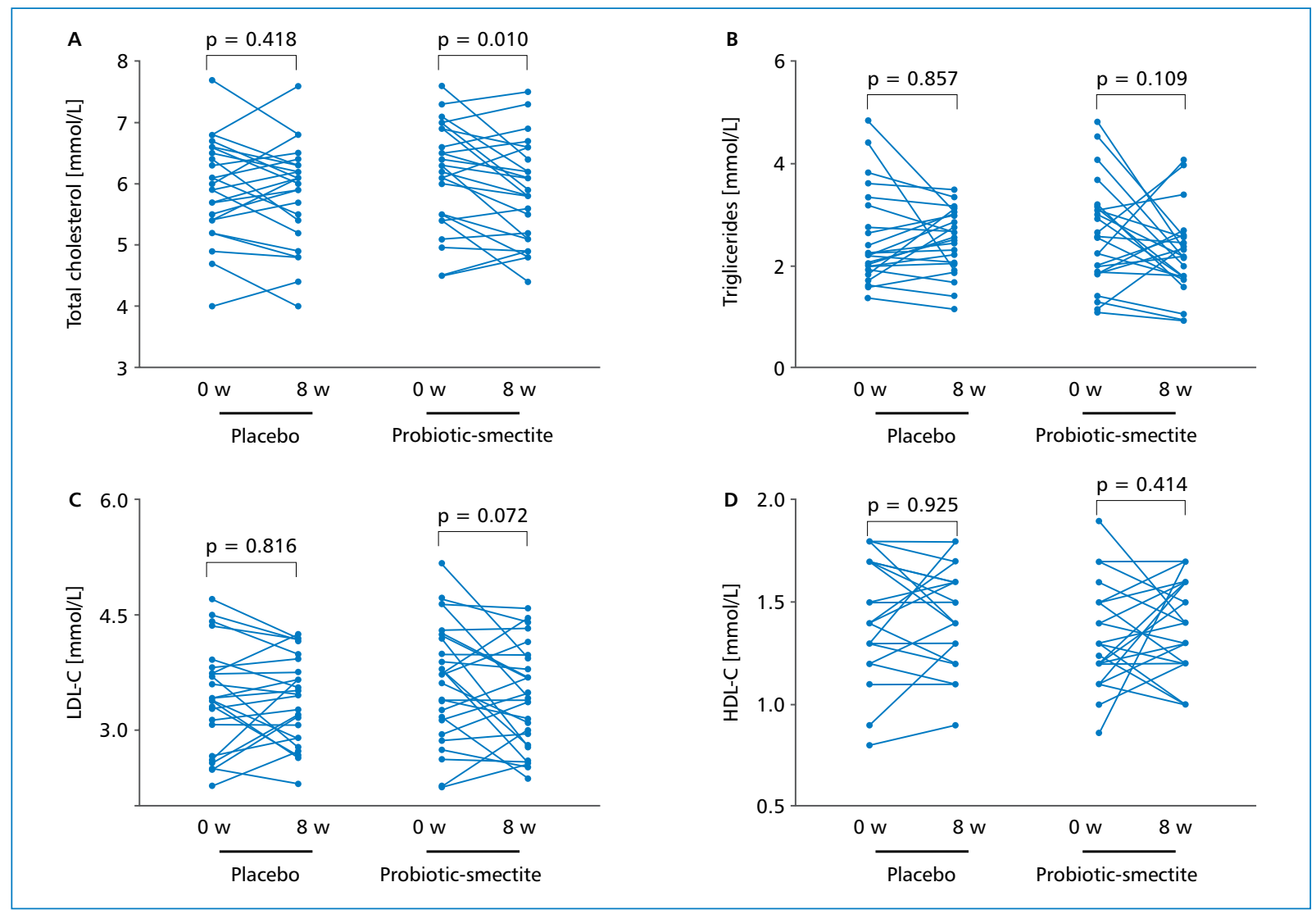

Figure 4. Secondary outcomes analysis with accent on lipid parameters. A, B, C, D - intra group analysis of changes at baseline and after interventon. Data expreesed as individual values at baseline and 8-week

ever, the mean reduction was significantly greater in the synbiotic group rather than in the placebo group $(p<0.001)$ [27].

Recent RCTs with similar design [21, 22] it has showed that administration of alive probiotic, alone or in combination with omega-3 fatty acids, had significant impact on hepatic fat content which characteristic reduction of FLI after intervention. No significant changes were noted LS measured with SWE in both trials [21, 22]. Moreover, co-administration of alive multi-strain probiotic mixture with omega-3 fatty acids once daily for 8 weeks to patients with NAFLD characterized with more pronounced changes in serum lipids and cytokines levels in secondary outcomes analysis as compared to probiotic only or probiotic-smectite, were we observed greatest reduction of transaminases activity. 


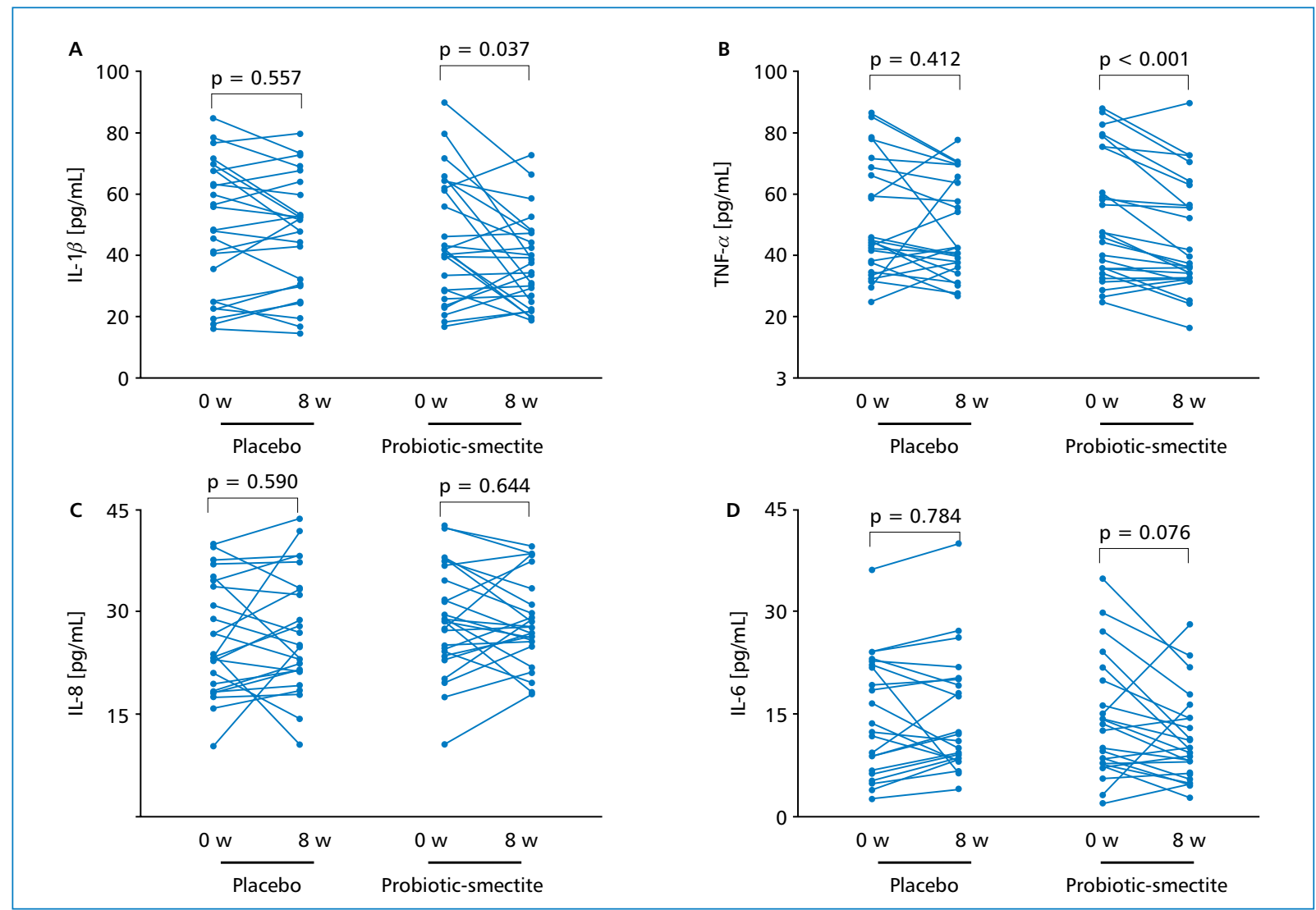

Figure 5. Secondary outcomes analysis with accent on cytokines changes. A, B, C, D - intra group analysis of changes at baseline and after interventon. Data expreesed as individual values at baseline and 8-week

Preparing RCT we have proposed that heartening of probiotic with smectite due to his ability support normal functional activity of intestinal mucosa, immunomodulating and cytoprotective effects, may lead to summation of their single positive effects. Smectite (bentonite) is a natural loamy poly-mineral which are formed by extremely small particles capable for hydration and demonstrate the most physiologically active properties in form of gel [28]. Smectite distributed on the intestinal tract surface and showed cytomucoprotective therapeutic effect by delivering energetic and plastic materials to epitheliocytes, improving the strength of the mucosa barrier and permitting mineral particles to interact with glycoproteins of the mucosa as well as with the microbial biolayer $[15,19]$. Another important property of smectite gel is direct absorbing capacity with viruses, toxins, radionuclides, heavy metals and bacterial endotoxins without "swallowing up" normal microbiota cells and physiologically important nutrients $[17,20]$. Following the mixing of probiotic biomass and smectite gel, the sorbent becomes bound to surface structures of bacterial cells and covers them with a protective layer promoting the increased probiotic biomass survival during its transit through the more aggressive gastrointestinal tract areas [19]. It is worth using nutraceuticals together with probiotics, as then possible to simultaneously improve mucosa cytoprotection and to restore its symbiosis with intestine physiological microflora.

In conclusion, in this RCT we confirmed previously reported animal data, that in NAFLD patients co-administration to, of probiotic with smectite due to his absorbent activity and stabilization mucus layer properties can impact on synergistic enhancement of single effect which manifested with reduction of LS, liver transaminases and chronic systemic inflammation.

The general limitations of our study were the use of the US technique instead of biopsy as the diagnostic criteria for NAFLD, the small sample size, and the absence of a longer term follow up. Therefore, modulation of the gut microbiota with probiotic and different nutraceuticals represents a new branch in NAFLD management, but further studies in larger cohorts are required to determine this beneficial effect.

\section{Conflicts of interest}

The authors report that they have no conflicts of interest. 
Table 2. Changes in secondary outcomes parameters between baseline and week 8 (M \pm SD)

\begin{tabular}{|c|c|c|c|}
\hline Parameters & Placebo group $(n=24)$ & Probiotic-smectite group $(n=26)$ & $\mathbf{P}$ \\
\hline \multicolumn{4}{|l|}{ ALT [IU/L] } \\
\hline Absolute value & $-0.37 \pm 6.55$ & $6.62 \pm 13.07$ & 0.022 \\
\hline Percentage from baseline & $-3.79 \pm 20.95$ & $10.32 \pm 32.1$ & 0.074 \\
\hline \multicolumn{4}{|l|}{ AST [IU/L] } \\
\hline Absolute value & $0.15 \pm 13.38$ & $3.31 \pm 6.88$ & 0.293 \\
\hline Percentage from baseline & $-7.59 \pm 29.93$ & $6.20 \pm 19.22$ & 0.056 \\
\hline \multicolumn{4}{|l|}{$\gamma$-GT [IU/L] } \\
\hline Absolute value & $1.23 \pm 10.27$ & $6.22 \pm 18.8$ & 0.256 \\
\hline Percentage from baseline & $-0.76 \pm 25.92$ & $-1.36 \pm 44.22$ & 0.954 \\
\hline \multicolumn{4}{|l|}{$\mathrm{TC}[\mathrm{mmol} / \mathrm{L}]$} \\
\hline Absolute value & $0.092 \pm 0.54$ & $0.295 \pm 0.53$ & 0.195 \\
\hline Percentage from baseline & $1.24 \pm 9.19$ & $4.39 \pm 8.55$ & 0.214 \\
\hline \multicolumn{4}{|l|}{ TG $[\mathrm{mmol} / \mathrm{L}]$} \\
\hline Absolute value & $0.03 \pm 0.79$ & $0.355 \pm 1.08$ & 0.236 \\
\hline Percentage from baseline & $-4.65 \pm 27.35$ & $5.408 \pm 41.79$ & 0.323 \\
\hline \multicolumn{4}{|l|}{ LDL-C [mmol/L] } \\
\hline Absolute value & $0.02 \pm 0.48$ & $0.230 \pm 0.62$ & 0.201 \\
\hline Percentage from baseline & $-1.04 \pm 15.99$ & $4.44 \pm 17.04$ & 0.247 \\
\hline \multicolumn{4}{|l|}{$\mathrm{HDL}-\mathrm{C}[\mathrm{mmol} / \mathrm{L}]$} \\
\hline Absolute value & $0.004 \pm 0.21$ & $-0.46 \pm 0.28$ & 0.435 \\
\hline Percentage from baseline & $-1.28 \pm 16.57$ & $-6.27 \pm 25.75$ & 0.424 \\
\hline \multicolumn{4}{|l|}{ TNF- $\alpha[\mathrm{pg} / \mathrm{mL}]$} \\
\hline Absolute value & $2.35 \pm 13.78$ & $6.75 \pm 7.73$ & 0.166 \\
\hline Percentage from baseline & $-0.71 \pm 33.65$ & $12.17 \pm 14.4$ & 0.081 \\
\hline \multicolumn{4}{|l|}{$\mathrm{IL}-1 \beta[\mathrm{pg} / \mathrm{mL}]$} \\
\hline Absolute value & $1.07 \pm 8.87$ & $6.74 \pm 15.59$ & 0.125 \\
\hline Percentage from baseline & $-1.65 \pm 22.13$ & $6.00 \pm 33.0$ & 0.345 \\
\hline \multicolumn{4}{|l|}{ IL-6 [pg/mL] } \\
\hline Absolute value & $-0.279 \pm 4.93$ & $2.28 \pm 6.3$ & 0.117 \\
\hline Percentage from baseline & $-18.11 \pm 43.72$ & $-5.19 \pm 94.2$ & 0.542 \\
\hline \multicolumn{4}{|l|}{ IL-8 [pg/mL] } \\
\hline Absolute value & $-0.78 \pm 7.06$ & $0.51 \pm 5.62$ & 0.472 \\
\hline Percentage from baseline & $-8.24 \pm 38.22$ & $-2.44 \pm 24.06$ & 0.521 \\
\hline \multicolumn{4}{|l|}{ IFN- $\alpha[\mathrm{pg} / \mathrm{mL}]$} \\
\hline Absolute value & $9.84 \pm 32.75$ & $21.37 \pm 50.33$ & 0.366 \\
\hline Percentage from baseline & $3.15 \pm 21.33$ & $4.31 \pm 32.95$ & 0.885 \\
\hline
\end{tabular}

\section{Authors" contributions}

N.K. and L.A. conceived and designed the study. N.K., G.M., L.B., T.F., L.K., D.K. and O.T. wrote the manuscript. All authors enrolled patients and approved the final version of the manuscript.

\section{Acknowledgements}

The authors express their sincere thanks to $\mathrm{Dr}$. Yankovsky Dmitro Stanislavovych for the help, advice and financial support of this work.

\section{REFERENCES}

1. Nascimbeni F, Pais R, Bellentani $S$, et al. From NAFLD in clinical practice to answers from guidelines. J Hepatol. 2013; 59(4): 859-871, doi: 10.1016/j.jhep.2013.05.044, indexed in Pubmed: 23751754.

2. Abenavoli L, Milic N, Di Renzo L, et al. Metabolic aspects of adult patients with nonalcoholic fatty liver disease. World J Gastroenterol. 2016; 22(31): 7006-7016, doi: 10.3748/wjg.v22.i31.7006, indexed in Pubmed: 27610012.

3. Bellentani S. The epidemiology of non-alcoholic fatty liver disease. Liver Int. 2017; 37 Suppl 1: 81-84, doi: 10.1111/liv.13299, indexed in Pubmed: 28052624. 
4. Musso G, Gambino R, Cassader M, et al. Meta-analysis: natural history of non-alcoholic fatty liver disease (NAFLD) and diagnostic accuracy of non-invasive tests for liver disease severity. Ann Med. 2011; 43(8): 617-649, doi: 10.3109/07853890.2010.518623, indexed in Pubmed: 21039302.

5. Mykhalchyshyn G, Kobyliak N, Bodnar P. Diagnostic accuracy of acyl-ghrelin and it association with non-alcoholic fatty liver disease in type 2 diabetic patients. J Diabetes Metab Disord. 2015; 14: 44, doi: 10.1186/s40200-015-0170-1, indexed in Pubmed: 25995986.

6. Kyriachenko Y, Falalyeyeva T, Korotkyi O, et al. Crosstalk between gut microbiota and antidiabetic drug action. World J Diabetes. 2019; 10(3): 154-168, doi: 10.4239/wjd.v10.i3.154, indexed in Pubmed: 30891151

7. World Health Organization and Food \& Agriculture Organization. Guidelines for the Evaluation of Probiotics in Food; Report of a Joint FAO/WHO Working Group on Drafting Guidelines for the Evaluation of Probiotics in Food; FAO/WHO: London, ON, Canada, 2002; Available online: ftp://ftp.fao.org/es/esn/food/ wgreport2.pdf.

8. Federico A, Dallio M, Godos J, et al. Targeting gut-liver axis for the treatment of nonalcoholic steatohepatitis: translational and clinical evidence. Transl Res. 2016; 167(1): 116-124, doi: 10.1016/j. trsl.2015.08.002, indexed in Pubmed: 26318867.

9. Wu TR, Lin CS, Chang CJ, et al. Gut commensal Parabacteroides goldsteinii plays a predominant role in the anti-obesity effects of polysaccharides isolated from Hirsutella sinensis. Gut. 2019; 68(2): 248-262, doi: 10.1136/gutjnl-2017-315458, indexed in Pubmed: 30007918

10. Cani PD, de Vos WM. Next-Generation beneficial microbes: the case of Akkermansia Muciniphila. Front Microbiol. 2017; 8: 1765, doi: 10.3389/fmicb.2017.01765, indexed in Pubmed: 29018410

11. Kobyliak N, Falalyeyeva T, Virchenko O, et al. Comparative experimental investigation on the efficacy of mono- and multiprobiotic strains in non-alcoholic fatty liver disease prevention. BMC Gastroenterol. 2016; 16: 34, doi: 10.1186/s12876-016-0451-2, indexed in Pubmed: 26976285.

12. Kobyliak N, Falalyeyeva T, Beregova T, et al. Probiotics for experimental obesity prevention: focus on strain dependence and viability of composition. Endokrynol Pol. 2017; 68(6): 659-667, doi: 10.5603/EP.a2017.0055, indexed in Pubmed: 29022648.

13. Kobyliak N, Falalyeyeva T, Bodnar P, et al. Probiotics supplemented with omega- 3 fatty acids are more effective for hepatic steatosis reduction in an animal model of obesity. Probiotics Antimicrob Proteins. 2017; 9(2): 123-130, doi: 10.1007/s12602-016-9230-1, indexed in Pubmed: 27660157.

14. Dupont C, Vernisse B. Anti-diarrheal effects of diosmectite in the treatment of acute diarrhea in children: a review. Paediatr Drugs. 2009; 11(2): 89-99, doi: 10.2165/00148581-200911020-00001, indexed in Pubmed: 19301931.

15. Weese JS, Cote NM, deGannes RVG. Evaluation of in vitro properties of di-tri-octahedral smectite on clostridial toxins and growth. Equine Vet J. 2003; 35(7): 638-641, indexed in Pubmed: 14649353.
16. Faure $C$. Role of antidiarrhoeal drugs as adjunctive therapies for acute diarrhoea in children. Int J Pediatr. 2013; 2013: 612403, doi: 10.1155/2013/612403, indexed in Pubmed: 23533446.

17. González R, de Medina FS, Martínez-Augustin O, et al. Antiinflammatory effect of diosmectite in hapten-induced colitis in the rat. Br J Pharmacol. 2004; 141(6): 951-960, doi: 10.1038/ sj.bjp.0705710, indexed in Pubmed: 14993105.

18. Mahraoui L, Heyman M, Plique $\mathrm{O}$, et al. Apical effect of diosmectite on damage to the intestinal barrier induced by basal tumour necrosis factor-alpha. Gut. 1997; 40(3): 339-343, doi: 10.1136/ gut.40.3.339, indexed in Pubmed: 9135522.

19. Kobyliak N, Falalyeyeva T, Boyko N, et al. Probiotics and nutraceuticals as a new frontier in obesity prevention and management. Diabetes Res Clin Pract. 2018; 141: 190-199, doi: 10.1016/j. diabres.2018.05.005, indexed in Pubmed: 29772287.

20. Kobyliak N, Abenavoli L, Falalyeyeva T, et al. Efficacy of probiotics and smectite in rats with non-alcoholic fatty liver disease. Ann Hepatol. 2018; 17(1): 153-161, doi: 10.5604/01.3001.0010.7547, indexed in Pubmed: 31072541.

21. Kobyliak N, Abenavoli L, Mykhalchyshyn G, et al. A multi-strain probiotic reduces the fatty liver index, cytokines and aminotransferase levels in NAFLD patients: evidence from a randomized clinical trial. J Gastrointestin Liver Dis. 2018; 27(1): 41-49, doi: 10.15403/jgld.2014.1121.271.kby, indexed in Pubmed: 29557414

22. Kobyliak N, Abenavoli L, Falalyeyeva T, et al. Beneficial effects of probiotic combination with omega-3 fatty acids in NAFLD: a randomized clinical study. Minerva Med. 2018; 109(6): 418-428, doi: 10.23736/S0026-4806.18.05845-7, indexed in Pubmed: 30221912.

23. Bedogni G, Miglioli L, Masutti F, et al. The Fatty Liver Index: a simple and accurate predictor of hepatic steatosis in the general population. BMC Gastroenterol. 2006; 6(5): 33, doi: 10.1186/1471-230X-6-33, indexed in Pubmed: 17081293.

24. Friedewald WT, Levy RI, Fredrickson DS. Estimation of the concentration of low-density lipoprotein cholesterol in plasma, without use of the preparative ultracentrifuge. Clin Chem. 1972; 18(6): 499-502, indexed in Pubmed: 4337382

25. Montandon SA, Jornayvaz FR. Effects of antidiabetic drugs on gut microbiota composition. Genes (Basel). 2017; 8(10), doi: 10.3390/ genes8100250, indexed in Pubmed: 28973971.

26. Wu H, Esteve E, Tremaroli V, et al. Metformin alters the gut microbiome of individuals with treatment-naive type 2 diabetes, contributing to the therapeutic effects of the drug. Nat Med. 2017; 23(7): 850-858, doi: 10.1038/nm.4345, indexed in Pubmed: 28530702.

27. Mofidi F, Poustchi H, Yari Z, et al. Synbiotic supplementation in lean patients with non-alcoholic fatty liver disease: a pilot, randomised, double-blind, placebo-controlled, clinical trial. $\mathrm{Br} \mathrm{J}$ Nutr. 2017; 117(5): 662-668, doi: 10.1017/S0007114517000204, indexed in Pubmed: 28345499.

28. Bailey SW. Summary of recommendations of AIPEA nomenclature committee on clay minerals. Clay Miner. 1980; 65: 1-7. 